\title{
Failure of Dexamethasone to Prevent Polymorphonuclear Leukocyte Infiltration During Experimental Acute Exudative Pyelonephritis and to Reduce Subsequent Chronic Scarring
}

Pascal R. Meylan and Michel P. Glauser

\author{
From the Division of Infectious Diseases, Department of \\ Internal Medicine, Centre Hospitalier Universitaire Vaudois, \\ Lausanne, Switzerland
}

\begin{abstract}
In experimental acute exudative pyelonephritis (AEP), a role for polymorphonuclear leukocyte (PMNL) infiltration in the pathogenesis of kidney scarring has been suggested indirectly. To directly quantitate PMNL infiltration during AEP, we developed an assay for measuring the content in the kidney of myeloperoxidase (MPO), an enzyme present in PMNLs and absent in kidney tissue. This assay was a specific and sensitive marker of the kidney PMNL content. We used this assay to measure in rats with AEP the effect of dexamethasone, administered in an attempt to mitigate the acute inflammatory response. Compared with saline, dexamethasone given during AEP strikingly reduced kidney swelling, measured by the kidney-weight increase, but failed to reduce PMNL infiltration, measured by the kidney MPO content. Despite reduced kidney swelling during AEP, dexamethasone treatment failed to prevent subsequent kidney scarring, an observation indicating that PMNLs play a role in the development of permanent kidney damage during AEP.
\end{abstract}

We have used a rat model of ascending unilateral obstructive pyelonephritis to investigate the relationship among the presence of bacteria in the kidney parenchyma (infection), the inflammatory processes that occur in response to infection, and the ensuing permanent kidney damage. In this model, acute exudative pyelonephyritis (AEP) leads to chronic pyelonephritis (CPN) with scarring and loss of kidney parenchyma [1-3]. We [4] and others [5-7] have previously shown that kidney scars might be prevented if the acute exudative processes were suppressed by early antibiotic therapy. In addition, drug-induced neutropenia aimed at reducing the PMNL infiltration during AEP afforded protection against CPN scars despite higher bacterial counts during acute infection $[8,9]$. These results were interpreted as indirect evidence that renal scarring (CPN) resulted

Received for publication 6 May 1987, and in revised form 13 October 1987.

Dr. Meylan was supported by grant 3.836.81 from the Swiss National Foundation for Scientific Research.

We thank Marlies Knaup for technical assistance, Sylvie Glasson for performing high-pressure liquid chromatography assays for dexamethasone, Dr. Angelika Delaloye-Bischof for help in radioisotopic studies, and Sylviane Bovey for typing the manuscript.

Please address requests for reprints to Dr. Michel P. Glauser, Division of Infectious Diseases, Department of Internal Medicine, Centre Hospitalier Universitaire Vaudois, 1011 Lausanne, Switzerland. from tissue damage due to excessive infiltration of PMNLs.

Because the acute inflammatory response appears to play a pivotal role in the pathogenesis of CPN, we tested the effect of dexamethasone administered during AEP on the PMNL infiltration (as measured by the myeloperoxidase [MPO] content of the kidneys) and on subsequent kidney scarring.

\section{Materials and Methods}

Preparation of rat exudate PMNLs. For preparing solubilized MPO, exudation of peritoneal PMNLs was elicited in rats as described by Baron and Proctor [10].

Extraction and assay of MPO. MPO was extracted and assayed as described by Bradley et al. [11], except that samples were first diluted 1:10 in lysis buffer.

Experimental design of dexamethasone therapy. Rats received four ip doses of either $1 \mathrm{~mL}$ of $0.9 \%$ $\mathrm{NaCl}$ or $1 \mathrm{~mL}$ of $0.9 \% \mathrm{NaCl}$ containing $0.5 \mathrm{mg}$ of dexamethasone sodium phosphate (Oradexon ${ }^{\circledR}$; Organon, Oss, the Netherlands), corresponding to 1.75 $\mathrm{mg} / \mathrm{kg}$ of dexamethasone. Injections were administered once a day at 8 A.M. over a four-day period. On the afternoon after the first injection, 230 rats were operated on in groups of 35-40 with AEP produced as previously described by using 06:K5:H1 [1, 
4]. Rats from each treatment group were randomly selected for killing at $1,2,3,7$, or $60 \mathrm{~d}$ after operation. Results of separate experiments were reproducible and were thus pooled for statistical analysis. Nine rats died during anesthesia; all surviving rats were included in the results.

Killing of animals and evaluation of effect of dexamethasone treatment. Rats were killed as described previously $[4,12]$. In each experiment the following parameters were evaluated: (I) The incidence of macroscopic pyelonephritis was tabulated. Kidneys that do not exhibit pyelonephritic lesions have been shown to harbor only low bacterial counts during the first few days after operation; these findings and can be considered to indicate failure to induce pyelonephritis $[4,12]$. (2) Bacterial counts were enumerated in kidney parenchyma $(\log \mathrm{cfu} / \mathrm{g}$ of kidney tissue). (3) The intensity of the acute inflammatory changes in the animals killed during AEP was assessed by the left/right kidney weight ratio (which provides a quantitative index of the severity of pyelonephritis and increases in proportion to suppuration during $\mathrm{AEP}$ ), and the kidney MPO content (which is calculated from the MPO activity in the kidney homogenates and takes into account the dilution due to homogenization and to the kidney weight). This parameter was used for estimating the intensity of PMNL infiltration. (4) The severity of kidney scarring in animals killed two months after operation was determined. During CPN the left/right kidney weight ratio decreases due both to destruction of the left kidney tissue and to compensatory hypertrophy of the right kidney [3].

As well as assessing the left/right kidney weight ratio as a measure of kidney destruction during CPN, we took advantage of the quantitative binding of technetium-99 dimercaptosuccinic acid to cortical tubular cells [13] to measure the remaining functional renal parenchyma. Six hours after iv injection of 125 $\mu \mathrm{Ci}$ of technetium-99 dimercaptosuccinic acid, rats with CPN were killed, and a $1-\mathrm{mL}$ sample of blood was drawn. The kidneys were excised and weighed, and radioactivity was counted by solid scintillation. The amount of circulating label in blood was negligible compared with that bound to kidneys. Thus, in addition to the left/right kidney weight ratio, a similar left/right kidney cpm ratio was computed.

Dexamethasone serum levels. Dexamethasone levels were determined in sera from five rats after ip injection of $0.5 \mathrm{mg}$ of dexamethasone sodium phosphate. Dexamethasone was assayed by high- performance liquid chromatography according to the method described by Lambert et al. [14].

Statistical analysis. Comparisons were done by the Wilcoxon rank-sum test. Relations between parameters were analyzed by the linear regression method. The incidence of pyelonephritis was compared by the $\chi^{2}$ test with Yates's correction. Statistical significance was defined as $P<.05$. All tests were two tailed.

\section{Results}

Activity of MPO and effect of normal kidney homogenate on the MPO assay. Normal kidneys contained very low levels of peroxidase activity. Furthermore, no peroxidase activity was detected in cultures (even when sonicated) of Escherichia coli O6:K5:H1.

The MPO activity recovered from peritoneal PMNL suspensions in $0.9 \% \mathrm{NaCl}\left(10^{6}-10^{8}\right.$ cells $\left./ \mathrm{mL}\right)$ was proportional to the number of PMNLs in each sample $(r=.995): 10^{6}$ PMNLs yielded $1.98 \pm 0.05$ (mean $\pm \mathrm{SE}$ ) U of MPO activity.

In testing whether normal kidney homogenate would interfere with the extraction or assay of MPO, samples of PMNL suspensions were centrifuged and resuspended in either $100 \mu \mathrm{L}$ of $0.9 \% \mathrm{NaCl}$ or 100 $\mu \mathrm{L}$ of pooled normal kidney homogenate containing a background level of $0.38 \mathrm{U}$ of peroxidase activity/mL of kidney homogenate. Normal kidney homogenate did interfere to some extent with the MPO assay: The MPO activity recovered from peritoneal PMNL aliquots mixed with normal kidney homogenate represented only $58.8 \% \pm 3.3 \%$ (mean \pm SE) of that recovered from peritoneal PMNLs suspended in $0.9 \% \mathrm{NaCl}$. However, the MPO activity recovered from $10^{6}-10^{8} \mathrm{PMNLs} / \mathrm{mL}$ suspended in normal kidney homogenate was proportional to the number of PMNLs in the specimen $(r=.992): 10^{6}$ PMNLs yielded $1.11 \pm 0.04$ (mean \pm SE) $\mathrm{U}$ of MPO activity.

Enzymatic properties of MPO from purified PMNLs and from pyelonephritic kidney homogenates. In contrast to the low level of peroxidase found in normal kidney homogenates, the peroxidase activity measured in homogenates of pyelonephritic kidneys during AEP increased considerably (up to $130 \mathrm{U} / \mathrm{mL}$ of kidney homogenate). The sensitivity to $\mathrm{pH}$ changes, heat, azide, and cyanide of this kidney peroxidase activity was similar to that of MPO from PMNLs harvested from peritoneal exudates (data not shown). 
Relation between kidney weight increase and kidney MPO content during AEP. A strong positive correlation existed between the MPO content and the left kidney-weight enlargement, expressed by the left/right kidney weight ratio ( $r=.82$ ), among 25 pyelonephritic rats killed three days after inoculation.

Correlation between morphological and radioisotopic assessment of kidney parenchymal loss (CPN scars). The relation between kidney parenchymal destruction and the reduced kidney weight ratio two months after operation was assessed in experiments using technetium-99 dimercaptosuccinic acid. Nineteen animals with pyelonephritis of varying severity demonstrated decreased radioactivity binding in the left kidney and corresponding increased binding in the right kidney, a finding showing that the compensatory hypertrophy of the right kidney was proportional to the destruction of the left kidney parenchyma. There was a very close linear relationship between the left/right kidney cpm ratio and the left/right kidney weight ratio: left/right kidney cpm ratio $=[1.04 \times($ left $/$ right kidney weight ratio) $]-$ 0.12 ( $r=.985$; cpm ratio range, $0.20-0.99$ ). These observations demonstrated therefore that the left/ right kidney weight ratio represents an accurate measure of the residual functional mass of the renal parenchyma during chronic scarring.

Effect of dexamethasone on bacterial counts and weight increase of the left kidney during AEP. Dexamethasone treatment had no significant effect on bacterial counts in the left kidney at any time at which animals were killed, in both animals with high bacterial counts that developed AEP and animals with low bacterial counts that did not exhibit pyelonephritic lesions (figure 1). Because exudative lesions developed only in animals with high bacterial counts in the left kidney, the effect of dexamethasone on the left/right kidney weight ratio was studied in this group of animals only. Compared with saline, dexamethasone treatment reduced by $56 \%$ the median increase in the left/right kidney weight ratio in animals killed at three days after bladder inoculation, the time of peak acute inflammatory response $(P<.001$; figure 2$)$. This effect disappeared in animals killed later during the course of AEP.

Effect of dexamethasone on MPO content of the left kidney. Figure 3 shows the MPO content of the left kidney of animals killed from one to seven days after operation. Rats with low bacterial counts in the left kidney and no macroscopic evidence of AEP had a MPO content of the left kidney similar

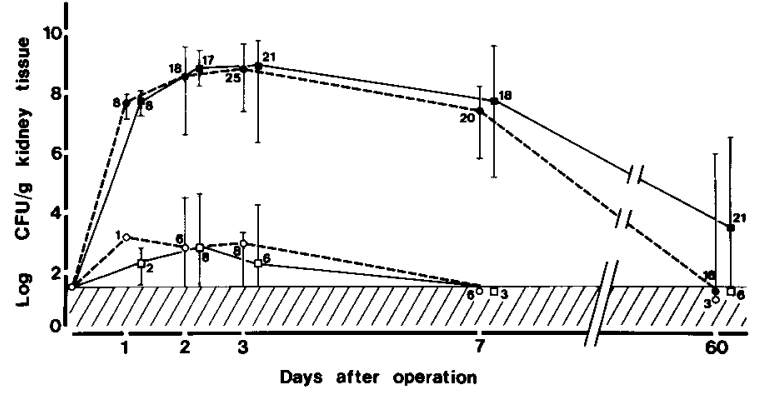

Figure 1. Effect of dexamethasone (squares) vs. control (circles) treatment on bacterial counts in the left kidney. The hatched area represents the counts below the threshold of bacterial detection. Data are median values; bars indicate ranges for each group. The number of animals in each group is given. Solid symbols represent animals with macroscopic evidence of pyelonephritis, these animals invariably had high bacterial counts in the left kidney; open symbols represent animals with low bacterial counts and without macroscopic evidence of pyelonephritis.

to that of normal kidneys throughout these intervals. Dexamethasone treatment did not influence the MPO content in these animals. In rats with high bacterial counts in the left kidney and macroscopic signs of AEP, dexamethasone treatment slightly but significantly reduced the MPO content of the left kidney in those rats killed early during the course of AEP, i.e., at $24 \mathrm{~h}(P=.04)$. However, no such difference was seen at later intervals, when the full development of the exudative response occurred and the MPO content increased considerably.

Effect of dexamethasone on severity of chronic pyelonephritis. Dexamethasone-treated animals killed two months after bladder inoculation had left/right kidney weight ratios similar to those of controls (figure 2). Thus despite the difference in the left/right kidney weight ratio in dexamethasonetreated and control animals during AEP, the extent of parenchymal loss two months later, measured by kidney weight changes, was similar in both treatment groups.

Dexamethasone serum level. Two hours after ip injection of $2.5 \mathrm{mg}$ of dexamethasone phosphate $/ \mathrm{kg}$, the mean \pm SD serum level of dexamethasone in five rats was $556 \pm 231 \mathrm{ng} / \mathrm{mL}$; values decreased to $119 \pm 60 \mathrm{ng} / \mathrm{mL}$ at $6 \mathrm{~h}$ and to $22 \pm 11 \mu \mathrm{g} / \mathrm{mL}$ at $12 \mathrm{~h}$.

\section{Discussion}

Several lines of evidence in the present experiments support the view that the MPO content of kidney 


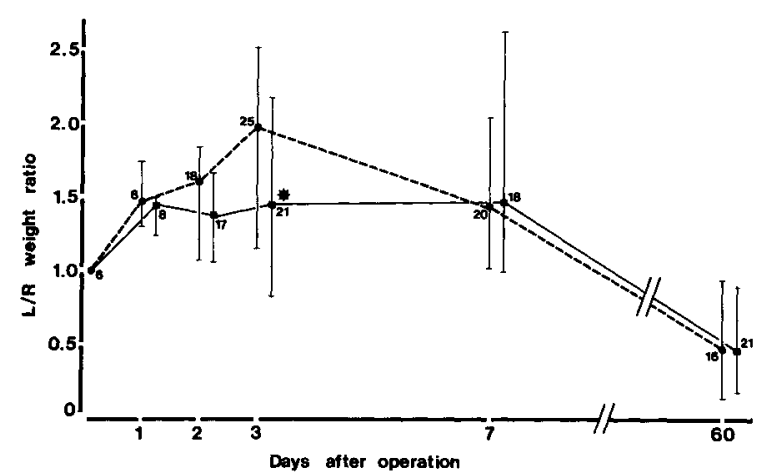

Figure 2. Effect of dexamethasone ( $\square$ ) vs. control ( $(\bullet)$ treatment on kidney swelling during AEP and on severity of scars during CPN, as reflected by the left/right $(L / R)$ kidney weight ratio. Data are median values; bars indicate ranges for each group. The number of animals in each group is given. The star denotes a highly significant $56 \%$ reduction $(P<.001)$ of the left/right ratio in dexamethasone-treated animals compared with controls killed three days after operation. Groups of animals that did not develop pyelonephritis are not shown here. Their left/right kidney weight ratio increased to 1.4 when they were killed $24 \mathrm{~h}$ after operation and decreased toward 1.0 in animals killed later.

homogenates can be used for quantitating the PMNL infiltration in kidney tissue. (I) Isolated rat PMNLs contained a large amount of MPO, whereas normal kidney homogenate did not. (2) Despite some interference of kidney homogenate with the MPO assay, the measure of the MPO activity was a quantitative measure of the number of PMNLs added to normal kidney homogenate samples. (3) The peroxidase activity in kidney homogenates increased in parallel with the macroscopic appearance of suppurative foci and the microscopic infiltration with PMNLs. (4) The peroxidase activity from pyelonephritic kidneys had enzymatic properties similar to the MPO activity extracted from purified PMNLs. Thus the kidney MPO content could be used as an index for tissue infiltration by PMNLs, which would allow the study of the effect of therapeutic interventions on the PMNL component of the inflammatory response.

The present data show that dexamethasone strikingly reduced the weight increase of the left kidney at the time of peak acute inflammatory response, that is, three days after operation. That dexamethasone had no significant effect on kidney weight at the earlier times of killing (one and two days after operation) is not surprising because the weight increase of the left kidney at this time is partly due

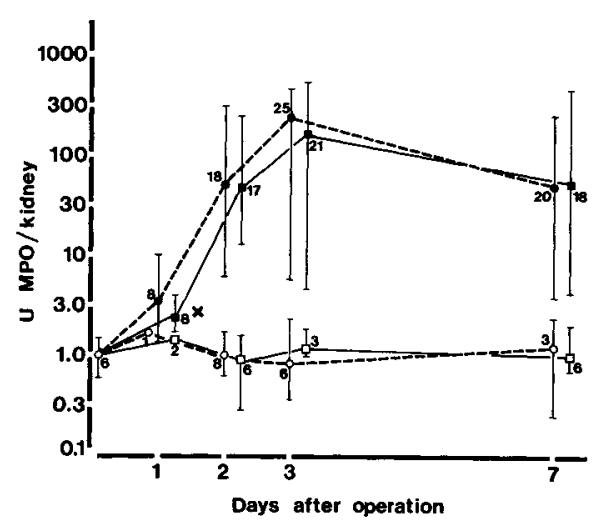

Figure 3. Effect of dexamethasone (squares) vs. control (circles) treatment on PMNL exudation, measured by the kidney MPO content. Data are median values; bars indicate ranges for each group. The number of animals in each group is given. Solid symbols represent animals with macroscopic evidence of pyelonephritis, these animals invariably had high bacterial counts in the left kidney; open symbols represent animals with low bacterial counts and without macroscopic evidence of pyelonephritis. The $X$ symbol denotes a slight but significant reduction $(P=$ .04) of the kidney MPO content in dexamethasone-treated rats killed one day after inoculation compared with controls. There was no such difference at any later interval.

to intrarenal hydronephrosis secondary to left ureteral ligation $[4,12]$. In contrast to its effect on the weight increase of the left kidney, dexamethasone failed to reduce the kidney infiltration by PMNLs, a finding suggesting that dexamethasone acted on the kidney weight by reducing the plasma exudation, not cellular migration into kidney tissue. This failure to reduce PMNL infiltration during AEP was accompanied two months after operation by a failure to prevent kidney scarring.

The lack of effect of dexamethasone on PMNL infiltration was not due to an inadequate dosage, because the peak serum levels of dexamethasone in the rats were five to 10 times higher than those achieved in humans after standard therapeutic doses of 0.1 $\mathrm{mg} / \mathrm{kg}$ [15-17]. Furthermore, the schedule of administration (once a day) seemed appropriate because dexamethasone was still detectable in serum $12 \mathrm{~h}$ after injection and because the half-life of the biologic effects of dexamethasone has been shown to be about twice that of the serum levels [17]. Indeed, the dexamethasone treatment schedule used here led to a potent glucocorticoid effect in the rats, as demonstrated by both the shrinkage of the spleen observed in dexamethasone-treated rats (data not shown), a typical glucocorticosteroid effect due to 
lymphocytic depletion [18], and the reduction of the weight increase of the left kidney during AEP.

Studies investigating the effects of pharmacological doses of glucocorticosteroids on the in vivo PMNL migration have led to conflicting results in several experimental systems and demonstrate either a slight reduction or no effect at all [19-26]. These apparently conflicting observations may result from differences with respect to the properties of each animal model used in testing the inflammatory response, to the characteristics and dosages of the glucocorticosteroid used, and to the schedule of administration. This latter point might be particularly relevant because the onset of the hormonal effect of corticosteroid treatment is usually delayed for several hours [15]. Our experiments differ in three respects from most of the observations mentioned above. (l) PMNL migration occurred in our experiments in response to the rapid development of high bacterial counts rather than in response to chemical or immunologic stimuli. (2) The PMNL infiltrates accumulating in the kidney parenchyma were quantified over several days, in contrast to observation periods of no more than $24 \mathrm{~h}$ in all of the above-mentioned studies. In our experiments, a reduction of PMNL migration by dexamethasone was also observed very early $(24 \mathrm{~h})$ during the development of AEP, but this effect vanished with the increasing inflammatory response, an observation raising questions about the biologic significance of this early reduction of PMNL infiltration. (3) Our model allowed accurate assessment of the permanent deleterious effect of PMNL infiltration on the kidney parenchyma. Here dexamethasone failed to reduce PMNL infiltration during AEP, and the subsequent development of kidney scars was not prevented. This result suggests that dexamethasone also failed to prevent the release of cytotoxic inflammatory mediators by stimulated PMNLs [27]. Because we have previously shown that PMNLs play an important role in the control of bacterial multiplication during AEP [8], the present observation that dexamethasone treatment did not influence bacterial counts is additional indirect evidence that dexamethasone did not reduce the activation and bactericidal effect of PMNLs on ingested $E$. coli and confirms that PMNLs are relatively refractory to glucocorticosteroid treatment [28].

In conclusion, we showed that measurement of the MPO activity in kidney homogenates can be used for estimating the PMNL infiltration of the kidney parenchyma. When administered during AEP, dexa- methasone only reduced the swelling of the pyelonephritic kidneys, not the PMNL infiltration. Therefore the anti-inflammatory effect of dexamethasone may not necessarily be linked to a reduced migration of phagocytes toward the inflammatory site, in contrast to a widely held belief [28]. Furthermore, dexamethasone failed to protect the rats from pyelonephritic scarring, a finding suggesting that dexamethasone was not able to reduce the cytotoxic activity of stimulated PMNLs. These data suggest that even a striking effect of glucocorticosteroids on the classic signs of inflammation (i.e., tumor, in the present study) is not necessarily linked to reduced tissue damage (functio laesa).

\section{References}

1. Brooks SJD, Lyons JM, Braude AI. Immunization against retrograde pyelonephritis. I. Production of an experimental model of severe ascending Escherichia coli pyelonephritis without bacteremia in rats. Am J Pathol 1974;74:345-58

2. Brooks SJD, Lyons JM, Braude AI. Immunization against retrograde pyelonephritis. II. Prevention of retrograde Escherichia coli pyelonephritis with vaccines. Am J Pathol 1974;74:359-64

3. Brooks SJD, Lyons JM, Braude AI. Immunization against retrograde pyelonephritis. III. Vaccination against chronic pyelonephritis due to Escherichia coli. J Infect Dis 1977;136:633-9

4. Glauser MP, Lyons JM, Braude AI. Prevention of chronic experimental pyelonephritis by suppression of acute suppuration. J Clin Invest 1978;61:403-7

5. Miller T, Phillips S. Pyelonephritis: the relationship between infection, renal scarring, and antimicrobial therapy. Kidney Int 1981;19:654-62

6. Slotki IN, Asscher AW. Prevention of scarring in experimental pyelonephritis in the rat by early antibiotic therapy. Nephron 1982;30:262-8

7. Ransley PG, Risdon RA. Reflux nephropathy: effects of antimicrobial therapy on the evolution of the early pyelonephritic scar. Kidney Int 1981;20:733-42

8. Bille J, Glauser MP. Protection against chronic pyelonephritis in rats by suppression of acute suppuration: effect of colchicine and neutropenia. J Infect Dis 1982;146:220-6

9. Shimamura T. Mechanisms of renal tissue destruction in an experimental acute pyelonephritis. Exp Mol Pathol 1981;34:34-42

10. Baron EJ, Proctor RA. Elicitation of peritoneal polymorphonuclear neutrophils from mice. J Immunol Methods 1982; 49:305-13

11. Bradley PP, Priebat DA, Christensen RD, Rothstein G. Measurement of cutaneous inflammation: estimation of neutrophil content with an enzyme marker. J Invest Dermatol 1982;78:206-9

12. Glauser MP, Francioli PB, Bille J, Bonard M, Meylan P. Effect of indomethacin on the incidence of experimental Escherichia coli pyelonephritis. Infect Immun 1983;40: 529-33 
13. Taylor A Jr. Quantitation of renal function with static imaging agents. Semin Nucl Med 1982;12:330-44

14. Lambert WE, De Slypere JPM, Jonckheere JA, Vermeulen A, De Leenheer AP. Improved liquid chromatographic determination of serum cortisol with double internal standardization compared to radioimmunoassay and fluorometry, and evaluated by isotope dilution/mass spectrometry. Anal Biochem 1983;134:216-23

15. Haynes RC Jr, Murad F. Adrenocorticotropic hormone; adrenocortical steroids and their synthetic analogs; inhibitors of adrenocortical steroid biosynthesis. In: Gilman Goodman A, Goodman LS, Rall TW, Murad F, eds. Pharmacological basis of therapeutics. New York: Macmillan, 1985:1459-89

16. Hare LE, Yeh KC, Ditzler CA, McMahon FG, Duggan DE. Bioavailability of dexamethasone. II. Dexamethasone phosphate. Clin Pharmacol Ther 1975;18:330-7

17. Meikle AW, Tyler FH. Potency and duration of action of glucocorticoids. Effects of hydrocortisone, prednisone and dexamethasone on human pituitary adrenal function. Am J Med 1977;63:200-7

18. Claman HN. Corticosteroids and lymphoid cells. N Engl J Med 1972;287:388-97

19. Meier R, Ecklin B. Die Wirkung des Hydrocortisons auf die infektionsbedingte lokale Leukozytenansammlung. Experientia 1960;16:204-5

20. Ackerman N, Martinez S, Thieme T, Mirkovich A. Relationship between adherence, chemotaxis and the accumulation of rat polymorphonuclear leukocytes at an inflammatory site. J Pharmacol Exp Ther 1982;221:701-7

21. Miyasaka K, Mikami T. Comparison of the anti-inflammatory effect of dexamethasone, indomethacin and BW 755C on carrageenin-induced pleurisy in rats. Eur J Pharmacol 1982;77: 229-36

22. Tarayre JP, Lauressergues $H$. Comparison of the effect of phenylbutazone, desonide and cyclophosphamide on four types of experimental pleurisy. J Pharm Pharmacol 1980; 32:408-12

23. Perper RJ, Sanda M, Chinea G, Oronsky AL. Leukocyte chemotaxis in vivo. II. Analysis of the selective inhibition of neutrophil or mononuclear cell accumulation. J Lab Clin Med 1974;84:394-406

24. Issekutz AC. Comparison of the effects of glucocorticoid and indomethacin treatment on the acute inflammatory reaction in rabbits. Immunopharmacology 1983;5:183-95

25. Almeida AP, Bayer BM, Horakova Z, Beaven MA. Influence of indomethacin and other anti-inflammatory drugs on mobilization and production of neutrophils: studies with carrageenan-induced inflammation in rats. J Pharmacol Exp Ther 1980;214:74-9

26. Täuber MG, Khayam-Bashi H, Sande MA. Effects of ampicillin and corticosteroids on brain water content, cerebrospinal fluid pressure, and cerebrospinal fluid lactate levels in experimental pneumococcal meningitis. J Infect Dis 1985;151:528-34

27. Weissman G, Smolen JE, Korchak HM. Release of inflammatory mediators from stimulated neutrophils. N Engl J Med 1980;303:27-34

28. Fauci AS, Dale DC, Balow JE. Glucocorticosteroid therapy: mechanisms of action and clinical considerations. Ann Intern Med 1976;84:304-15 